\title{
Tangence
}

\section{La madeleine revisitée : Les champs d'honneur de Jean Rouaud}

\section{Hélène Gaudreau}

Numéro 52, septembre 1996

Tours et détours du romanesque : Minuit aujourd'hui

URI : https://id.erudit.org/iderudit/025917ar

DOI : https://doi.org/10.7202/025917ar

Aller au sommaire du numéro

Éditeur(s)

Tangence

ISSN

0226-9554 (imprimé)

1710-0305 (numérique)

Découvrir la revue

Citer cet article

Gaudreau, H. (1996). La madeleine revisitée : Les champs d'honneur de Jean

Rouaud. Tangence, (52), 65-76. https://doi.org/10.7202/025917ar d'utilisation que vous pouvez consulter en ligne.

https://apropos.erudit.org/fr/usagers/politique-dutilisation/ 


\section{La madeleine revisitée : Les champs d'honneur de Jean Rouaud}

\section{Hélène Gaudreau}

La critique journalistique dit principalement des romans de Jean Rouaud qu'ils sont de "la mémoire ". C'est résolument placer Les champs d'bonneur et Des bommes illustres, publiês chez Minuit, dans le sillage de l'œuvre de Proust. Si l'auteur de la Recberche annonce mieux que quiconque en France l'évolution que connaîtra le roman au $x^{\mathrm{e}}$ siècle, il n'en reste pas moins infiniment loin du Nouveau Roman et des recherches formelles menées par les Butor, Pinget, Robbe-Grillet et autres nouveaux romanciers, publiés en grande partie par les éditions de Minuit dans les années cinquante et soixante. On sait que la maison d'édition a gardé, encore aujourd'hui, un lien essentiel avec la modernité romanesque, à laquelle l'œuvre de Rouaud ne semble pas correspondre en tous points. Il est vrai que d'autres romans publiés chez Minuit ne sont pas nécessairement de facture avantgardiste, mais la thématique tournée vers le passé privilégiée par Rouaud accentue ce que l'on pourrait appeler le caractère classique de son écriture.

Les champs d'bonneur, récit dans l'ensemble consacré à la famille du père du narrateur, commence par dessiner le portrait du grand-père maternel. Celui-ci est le dernier de la série ${ }^{1}$ des disparus, nous dit-on, celui des morts qui seront évoqués dont le souvenir est le plus récent; il apparaît dès lors comme naturel que s'ouvre sur lui un récit à rebours qui semble simplement remonter le flot des souvenirs comme ils se présentent. C'est ainsi que la narration rappelle ensuite à la mémoire une petite tante, morte elle aussi, peu de temps après son neveu, le père du narrateur, dont elle demeurait la seule famille. Et la liste des disparus

1 . C'est grand-père qui a clos la série, manière d'enfoncez-vous-bien-ça-dansla-tête tout à fait inutile. Jean Rouaud, Les champs d'bonneur, Paris, Minuit, 1990 , p. 9. Pour éviter de multiplier les notes, les nombreuses références à ce roman seront données ainsi dans la suite du texte: $C H$ suivi du numéro de la page; les quelques références à Des bommes illustres (Paris, Minuit, 1993) seront indiquées par $H I$, suivi du numéro de la page. 
se poursuit, se prolonge et, comme par associations d'idées, remonte le cours du temps, avec le récit actualisé des circonstances entourant la mort des frères de la tante, fauchés à la guerre, très longtemps avant la "série" précédente. Le lien thématique est assuré par la confusion mentale de la tante Marie qui, l'homonymie aidant, confondait la mort de son neveu Joseph avec celle de son frère ${ }^{2}$. De fil en aiguille, nous accompagnerons également au cimetière les grands-parents paternels du narrateur, ce dont est témoin, alors qu'il était encore jeune, le grand-père maternel sur qui s'est ouvert le récit. La boucle est ainsi bouclée, le cercle refermé, le temps en quelque sorte retrouvé. Et le récit semble dès lors porté par une logique qui n'est apparemment rien d'autre que thématique, celle de la mort, puisque - le secret de toute vie s'abreuve à cette source noire. ( $\mathrm{CH}, \mathrm{p}$. 42). Une telle matière n'est pas propice, en apparence du moins, aux constructions géométriques ou intellectuelles communément associées au Nouveau Roman et dont on s'attend à ce qu'elles repoussent sans cesse les limites formelles du genre.

En effet, l'écriture de Rouaud, un dosage délicat d'ironie et de sensibilité, d'érudition et d'anecdote, de culture et d'humour, se déploie en longues phrases souples et rythmées, sans bouleverser systématiquement l'horizon d'attente du lecteur. De facture classique, sa prose recourt abondament à l'image et à la comparaison; et si les descriptions sont nombreuses, précises et détaillées, si elles font appel à un vocabulaire spécialisé, elles

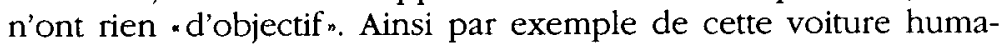
noïde :

La 2 CV est une boîte crânienne de type primate: orifices oculaires du pare-brise, nasal du radiateur, visière orbitale des pare-soleil, mâchoire prognathe du moteur, convexité pariétale du toit, rien n'y manque, pas même la protubérance cérébelleuse du coffre arrière ( $\mathrm{CH}$, p. 34).

On y sent moins le désir d'exhaustivité, la volonté d'obliger le lecteur à reconstituer l'objet - caractéristique de nombreux nouveaux romanciers --, que le plaisir de donner à voir la chose décrite d'une façon inusitée. De plus, les personnages ressuscités

2 Cette homonymie pourrait faire penser à The Sound and Fury de Faulkner, mais l'effet n'est pas du tout du même ordre : chez Rouaud, c'est un personnage qui est victime de confusion mentale et non le lecteur qui est confondu par une subversion textuelle. 
par la plume de Rouaud ont une identité dont le profil psychologique est bien dessiné et ils procèdent d'une généalogie; ils n'ont rien en commun avec ces * supports de hasard ${ }^{4}$ à peine identifiés par des pronoms, vagues silhouettes suggérées par les romanciers de l'école du regard, qui refusaient que l'intrigue et les personnages volent la vedette à l'écriture et résistaient à l'illusion référentielle.

Bien que les nouveaux romans de Minuit soient en général moins * éclatés - que leurs prédécesseurs, la facture des récits de Rouaud demeure donc au premier abord beaucoup plus classique que celle des romans auxquels nous ont habitués la plupart des autres romanciers publiés aujourd'hui par cette maison désormais associée au Nouveau Roman. Minuit accueille le plus souvent en effet les écritures minimalistes et qualifiées, à tort ou à raison, de cérébrales des Jean-Philippe Toussaint, Tropmann, Jean Echenoz, etc., dont les romans aux descriptions géométriques extrêmement précises, quasi techniques, mettent en scène un petit nombre de silhouettes urbaines qui vivent, généralement au présent et dans un univers dépersonnalisé, des situations dont le sens leur échappe.

Dès lors, on peut se demander comment ce récit qui témoigne d'une origine, comment cette quête d'un narrateur tourné vers le passé (et pourtant paradoxalement absent, j'y reviendrai) est assimilable à la littérature d'avant-garde; pourquoi ce roman où foisonnent les portraits de personnages pittoresques et provinciaux se retrouve à le même enseigne que les précédents.

Ces questions se résolvent pourtant d'elles-mêmes à la faveur d'une lecture plus attentive: chez Rouaud, la complexité syntaxique de la phrase, la saveur de l'anecdote et la richesse des images et du vocabulaire éclipsent d'abord une logique structurelle, une architecture proprement textuelle, qui trace les contours de la véritable aventure de ce roman, celle d'une écriture (pour reprendre la désormais célèbre formule de Ricardou). L'histoire - si tant est qu'il y en ait une - est en effet ici celle de quelqu'un qui écrit, à partir de traces écrites dont il essaie de reconstituer la genèse. Ricardou est ainsi pris au mot, à la lettre,

3 Nathalie Sarraute, - Ce que je cherche à faire, dans Nouveau Roman: bier, aujourd'but, tome 2, Pratiques, Paris, Union générale d'édition, coll. «10/18*, p. 26. 
dans un sens thématique qu'il n'avait sans doute pas prévu, un sens qui renvoie d'abord au contenu, quoi que la forme y contribue également. Les textes de Rouaud sont en effet pétris de descriptions focalisées qui s'attardent longuement à de menus événements, creusent profondément une matière romanesque minimale: chacun des récits pourrait se comparer à une série de tableaux sans véritable intrigue. Le chapitre consacré à la pluie sous toutes les formes qu'elle prend en pays nantais est un exemple représentatif de cette écriture qui se déplie en longues digressions et ne se justifie que d'elle-même.

Qu'il pleuve à marée montante, ce n'est pas à proprement parler une pluie. C'est une poudre d'eau, une petite musique méditative, un hommage à l'ennui. Il y a de la bonté dans cette grâce avec laquelle elle effleure le visage, déplie les rides du front, les repose des pensées soucieuses. Elle tombe discrète, on ne l'entend pas, ne la voit pas, les vitres ne relèvent pas son empreinte, la terre l'absorbe sans dommage ( $\mathrm{CH}, \mathrm{p} .21)$.

Mais plus encore que par l'évident plaisir que prend le narrateur à trouver les formules inédites et les rapports de cause à effet singuliers ${ }^{4}$, c'est par sa structure même que Les champs d'bonneur est une entreprise scripturaire - aux deux sens du mot! - avant que d'être narrative.

Ce sont en effet des traces écrites - non seulement matérielles, signes tracés sur une page, mais aussi littéraires, références à d'autres textes, dont la Bible - qui sont à la source du récit, qui se donnent dans le texte, quand on y regarde de plus près, comme son origine. Cette structure se concrétise en une série de contrepoints thématiques et de signifiants récurrents qui non seulement traversent tous les chapitres, mais assurent l'unité de chacun d'eux; ce qui n'est pas sans rappeler la notion de métaphore structurelle proposée par Ricardou, "par laquelle un texte se construit et, spécifiquement, fonctionne ${ }^{5}$.

4 En voici un exemple, parmi d'autres: "Grâce à quoi [la pluie] les opticiens font des affaires. Non que les myopies soient ici plus répandues qu'ailleurs, mais nettoyer ses verres avec un pan de chemise sorti en catimini du pantalon, avec un coin de nappe au restaurant ou l'angle intact d'un mouchoir roulé en boule au creux de la main, le risque se multiplie que les lunettes se démantibulent, tombent et se brisent * ( $C H$, p. 17).

5 Notion qu'il illustre d'ailleurs avec un exemple choisi chez Proust. Jean Ricardou, Problèmes du Nouveau Roman, Paris, Éditions du Seuil, 1967, coll. - Tel Quel•, p. 136. 
En l'occurrence et fort significativement, la logique inhérente à la composition des Champs d'bonneur repose en grande partie sur la métaphore la plus proustienne qui soit, celle d'une madeleine trempée dans le thé comme vecteur de la résurrection du passé; elle est métonymiquement représentée à son tour dans le récit de Rouaud par la commune de Commercy, spécialisée, comme le précise le dictionnaire encyclopédique cité dans le texte, dans la fabrication des gâteaux en forme de coquille. La madeleine en acquiert une valeur de signifiant, également convoquée par l'entreprise du narrateur, proprement résurrectionnelle, valeur renforcée à deux reprises dans le texte par une interprétation de la thématique pascale où Marie-Madeleine ${ }^{6}$, première à constater la disparition du corps du Christ le jour de la résurrection, joue un rôle fondamental rappelé par le narrateur; lequel, incidemment, en profite pour se désigner de façon détournée:

Que dit Jean sur la réapparition de Jésus ce matin halluciné où achoppe le salut de la multitude? Que Marie-Madeleine, le jour à peine levé, accourt au tombeau et le trouve vide - MarieMadeleine l'amoureuse effrénée qui couvrait d'un coûteux parfum de Galilée les pieds du marcheur sublime en les oignant de ses cheveux. Elle demande à celui qu'elle prend pour le gardien du jardin où l'on a déposé le corps du supplicié, car elle désire l'emporter, l'assurer par-delà la mort de la pérennité de son amour. Marie-Madeleine ne se lamente pas qu'on l'ait trompée au sujet de la résurrection, elle ne joue pas l'offensée, ne se calfeutre pas dans l'espérance d'une amnistie, honteuse qu'on ait ainsi abusé de sa crédulité, elle se moque du qu'en-dira-t-on qui paralyse les apôtres. Cette révélation de l'amour lui suffit, l'occupera jusqu'à la fin de ses jours ( $\mathrm{CH}$, p. 115-116, c'est moi qui souligne).

Traverser la clôture du texte pour identifier l'instance énonciatrice de ce récit au prénom qui figure sur la couverture du livre, celui de l'auteur, est une audace que je ne me serais pas permise si le narrateur lui-même n'avait confirmé le rapprochement dans Des bommes illustres:

Comme le lendemain 27 décembre est la Saint-Jean l'Évangéliste, il n'oublie pas en vous embrassant de vous souhaiter votre fête. Il vous fait un peu peur cet homme, bien qu'il n'ait jamais

6 Ce prénom contient également celui de la tante Marie, occupée toute sa vie elle aussi de l'amour du Christ. C'est d'ailleurs à ce personnage que la thématique pascale est directement reliée. 
porté la main sur vous, mais son autorité en impose et vous cloue souvent le bec, alors quelle tête ferez-vous quand vous apprendrez, des années plus tard, qu'il vous a donné ce prénom-là, fêté à cette date-là, parce que c'est celui que portait le disciple bien-aimé? [...] Beaucoup plus tard encore il vous viendra à l'esprit que c'est aussi celui-là, le préféré, qui a rendu compte: :c'est ce disciple qui témoigne au sujet de ces choses et qui les a écrites - (HI, p. 106-107).

Du coup, c'est toute l'entreprise romanesque qui serait à revoir à la lumière de cette constatation qui donne encore plus de force à l'onomastique biblique qu'on y observe. Mais nous suivons pour l'instant une autre piste, celle non pas du pourquoi mais du comment de l'écriture.

Le signifiant madeleine prolonge ses possibilités associatives avec l'humble grotte magdalénienne bricolée par une jeune fermme (une grand-tante du narrateur), un Noël de guerre (nativité qui prolonge le retentissement de la thématique pascale, sa contrepartie), alors qu'elle vient de donner à son mari, revenu du front pour l'occasion, un fils qu'il ne verra pas grandir. Nous apprendrons plus tard que cet homme est au centre d'un "mystère" relié au village producteur de madeleines, Commercy. La métaphore est ainsi filée pour revenir en quelque sorte à son point de départ: la pâtisserie proustienne.

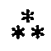

Cette résurrection d'un monde sur le point de sombrer dans l'oubli est bien sûr déjà un lien suffisant, bien que facile, pour faire de Rouaud un émule de Proust. Sans compter que la petite tante opiniâtre s'impose telle une Léonie devenue soudain débordante d'activités, mais plus que jamais dévote, et que la narration nous fait traverser, dans un rythme lent de promenade, des paysages minutieusement décrits. Mais il n'y aurait pas d'intérêt à poursuivre au-delà la recherche d'une correspondance qui, tout compte fait, pourrait se retrouver dans l'œuvre de nombre d'écrivains, si une saveur particulière n'était donnée comme propice aux réminiscences - et de là source possible d'écriture:

[Grand-père] rapportait un succulent fromage de labbaye, à la croûte crème orangée, pâte jaune paille piquée de trous d'épingles, ferme et moelleux - qui doit appartenir au rayon des 
saveurs oubliées, sans qu'on s'en émeuve faute de comparaison, mais envolés du même coup mille petits satoris délicieux ( $\mathrm{CH}$, p. 40).

L'ironie repérable ici - dont le "faute de comparaison" veut surtout souligner à mon avis l'humilité de la, classe sociale de ses personnages par rapport à ceux de la Rechercbe (ironie sur laquelle joue d'ailleurs le titre, qui donne en quelque sorte "les champs d'honneur" comme un euphémisme de "fosse communen) - démontre bien s'il le fallait que Rouaud n'est pas naïvement proustien, ou qu'il ne s'agit pas pour lui de suivre un modèle. Sans aller jusqu'à voir ici un pastiche, nous pouvons légitimement croire que les clins d'œeil participent pleinement de l'effet esthétique recherché, et témoignent d'un souci d'intertextualité.

En l'occurrence, la citation précédente prendra après-coup plus de saveur quand les désormais célèbres petits gâteaux qu'elle a rappelés à l'esprit du lecteur seront nommés, au détour d'une phrase et entre parenthèses, pour annoncer non sans humour la spécialité de la petite commune de Commercy, à proximité des champs de bataille de la Grande guerre pendant laquelle sont morts deux grands-oncles paternels auxquels la troisième partie du récit est consacrée.

Maintenant Joseph [homonyme du père du narrateur] annoncé mort - son nom sur une image pieuse et patriotique qui se vend $0 \mathrm{fr} 05$, pour les ceuvres, à la cure de Commercy (souspréfecture de la Meuse, spécialité de madeleines), sertie d'un mince bandeau noir, monument de tristesse à l'en-tête d'un titre de roman héroïque: :Les Champs d'Honneur * $(\mathrm{CH}, \mathrm{p} .162)$.

Il ne saurait y avoir de hasard dans cette conjonction où l'on retrouve, avec les allusions religieuse et littéraire, le titre même du récit. Pour ajouter au potentiel signifiant, la commune en question est liée à un mystère familial oublié - pour ainsi dire refoulé - réapparu inopinément dans le délire de la tante Marie:

Elle était notre marc de café infaillible dans la lecture du passé. Simplement, les réponses à nos questions arrivaient dans le désordre, et achoppaient finalement sur le mystère Commercy, comme si dans ce puzzle nous nous trouvions en présence d'une pièce en trop ( $\mathrm{CH}, \mathrm{p}$. 123).

Cette "pièce en trop" est en quelque sorte un tabou familial qui concerne l'un des oncles morts à la guerre (*une inhumation à la 
72

sauvette, comme un comédien" $\mathrm{CH}, \mathrm{p}$. 122). Les circonstances de cette situation honteuse sont tenues secrètes par ceux qui feignent d'avoir oublié, ce qui, bien évidemment, décuple l'importance du non-dit et l'entoure de cette aura de mystère qui pique la curiosité. L'énigme sera cependant résolue; c'est justement, mine de rien, l'entreprise du narrateur dont tous les fils discursifs ramènent à ce point de convergence pour former en quelque sorte le noud du récit. Mais celui qui mène la narration ne dispose pas que de sa seule mémoire, des sources écrites alimentent son imaginaire. Le grand-père maternel, venu à l'occasion de la mort du père du narrateur ("cet homme jeune qu'il aimait") et intrigué par cette histoire lacunaire, a réuni dans une boîte à chaussures des traces écrites reliées aux événements de Commercy, témoignages du passé qui ne pouvaient avoir de sens que vus à la lumière les uns des autres: photos, cahiers jaunis et cartes postales, documents qui ne suscitent sur le moment qu'un intérêt vite déçu.

Restait la boîte à chaussures. Cette passation solennelle, ces quelques mots secrètements murmurés, c'était clair: grand-père avait regroupé dans cé carton l'essentiel de ses trouvailles. On agita la boite, elle ne tinta pas. Ne contenant pas d'or, elle nous apporterait pour le moins la preuve de quelque ascendance glorieuse ( $\mathrm{CH}$, p. 140).

C'est à partir de ces documents que le narrateur peut, bien des années plus tard, reconstituer les événements reliés à Commercy, ce qui semble dès lors le véritable but des Champs d'bonneur, entreprise résolument placée dans la perspective d'une irréductible subjectivité.

Qu'y a-t-il à l'intérieur d'une noix? L'imagination s'emballe: la caverne d'Ali Baba? Le bois de la vraie Croix? La voix de Rudolf Valentino? On la casse et l'avale. On apprend qu'elle contient oligo-éléments et vitamines, glucides et lipides, mais que la caverne d'Ali Baba est dans la tête de Schéhérazade, le bois de la vraie Croix dans l'arbre de la Connaissance et la voix de Rudolf Valentino dans le regard du sourd ( $\mathrm{CH}, \mathrm{p} .141$ ).

La logique structurelle s'affirme donc dès l'incipit: le grandpère maternel a peut-être clos une série, bouclé un cycle, mais ce n'est pas sans d'abord avoir ouvert la voie par où la venue à l'écriture serait possible. Car, comme le souligne presque d'entrée de jeu le narrateur, "grand-père n'était pas loin, à portée de nos jeux quand on l'imaginait à l'autre bout de son âge dans un bric- 
à-brac de souvenirs anciens. $(C H$, p. 14). C'est lui, je l'ai dit, qui met à la portée du narrateur les pièces qui lui permettent de poursuivre le jeu amorcé par le délire de la tante, de trouver la solution du puzzle familial qu'elle avait involontairement remis en circulation. Il est donc doublement logique que ce soit sur la figure du vieil homme que s'ouvre le récit.

C'est en deux temps que le "mystère Commercy" a partie liée avec la mort et la mémoire. Si le narrateur peut nous dire que l'oncle Joseph est mort à la guerre, c'est que la sœur du défunt avait pris la peine d'écrire le nom de son frère sur la carte vendue pour les ouvres de Commercy "et ce court commentaire sauve Joseph de la longue nuit amnésique ( $\mathrm{CH}, \mathrm{p}$. 163). C'est d'abord ce frère avec lequel l'onomastique lui fait former un couple chastement incestueux - Joseph et Marie! - que la malade appelle dans son délire.

Mais le mystère ne concerne pas Joseph, dont la date et le lieu de la mort permettent au narrateur d'en imagniner les circonstances atroces, et de réactualiser l'effet sournoisement dévastateur des premières bombes chimiques. Joseph est surtout un jalon pour atteindre un nouveau personnages. C'est Émile qui, se sentant invincible après la mort de son frère, est allé se faire tuer à Commercy, véritable fosse commune qui ne rendra pas les corps de ceux qu'elle aura engloutis. Plus encore que son frère, Émile est donc menacé de se confondre dans l'oubli :

Les cadavres abandonnés s'enlisaient peu à peu dans la glaise, glissaient au fond d'en entonnoir, bientôt ensevelis sous une muraille de terre. On trébuchait pendant un assaut sur un bras à demi déterré, un pied, et, tombant le nez sur le nez d'un cadavre, on jurait entre ses dents - les siennes et celles du mort. C'était une fâcheuses invite, ces crocs-en-jambe sournois des trépassés. Mais on en profitait pour arracher autour du cou les plaques d'identité, sauver ces masses anonymes d'un futur sans mémoire, les ramener à l'état civil, comme si le drame du soldat inconnu était moins d'avoir perdu la vie que son nom. C'est sans doute ainsi qu'on avait annoncé à sa femme qu'Émile était mort, son corps enfoui dans le secteur des Hauts-de-Meuse (CH, p. 168).

L'absence de cadavre aux obsèques avait rendu la disparition d'Émile problématique et entretenu bien des espoirs fallacieux. Avait-il, frappé d'amnésie, refait sa vie dans un pays étranger? Le contexte est donc propice à semer le mystère. Quelque dix ans 
après la guerre, une lettre d'un camarade (également retrouvée dans la boite à chaussures), précisant le lieu de la tombe improvisée, apprenait à la famille qu'Émile avait échappé à la fosse commune: elle en confirmait du même coup le décès.

Pierre, le frère survivant, grand-père paternel du narrateur, ira récupérer la dépouille après réception de la lettre. Son entreprise est illicite et il convient de ne pas l'ébruiter, d'où le non-dit qui confirme le mystère. Pour ajouter aux déconvenues de la famille, c'est deux séries d'ossements que rendra la terre gelée et que, faute de pouvoir les distinguer l'une de l'autre, Pierre ramènera en toute illégalité dans sa voiture, emballées comme de la vaisselle dans des caisses destinées à distribuer des madeleines. L'expédition revêt alors un caractère honteux qui permet au mystère de s'épanouir et au tabou de prendre bouture. Pierre consigne néanmoins les faits dans le petit journal de voyage qu'il tient à l'intention d'Aline, sa femme bien-aimée, petit cahier noir qui témoigne également de cet immense amour, précieuse pièce à conviction qui sera retrouvée par le narrateur, comme les autres documents, dans la boîte à chaussures.

Rouaud a en quelque sorte opéré un renversement des valeurs, habilement construit une double subversion textuelle. En effet, la ville des madeleines, cette pâtisserie que la littérature associe désormais à la mémoire, est ici une fabrique de morts dont la mémoire est oblitérée par les euphémiques "champs d'honneur", champs qui ont englouti, réduit à l'anonymat, des centaines de soldats, pour qui le In memoriam n'est que vaines paroles. Mais, comme Marie-Madeleine au matin de Pâques, le narrateur veut rendre hommage à ceux qui sont morts. Comme l'Évangéliste, c'est par le témoignage, par l'écriture qu'il tire de l'oubli ce qui ne peut être ressuscité autrement. Car souvenonsnous que, dans l'écriture revisitée par Rouaud, le Christ est mort et que Dieu était du côté du Pharaon (des Allemands ${ }^{7}$ ).

Cette écriture aux propriétés renversantes se nourrit donc du contenu du carton à chaussures, essentiellement traces écrites ras-

7 . C'est ainsi que Joseph vit se lever une aube olivâtre sur la plaine d'Ypres. Dieu, ce matin-là, était avec eux. Le vent complice poussait la brume verte en direction des lignes françaises, pesamment plaquée au sol, grand corps mou épousant les moindres aspérités du terrain, s'engouffrant dans les cratères, avalant les bosses et les frises de barbelés, marée verticale comme celle en mer Rouge qui engloutit les chars de l'armée du pharaon. $(\mathrm{CH}, \mathrm{p} .155)$ 
semblées par le grand-père maternel à qui est consacrée la première partie du récit. L'ironie aidant, la modeste histoire familiale est devenue une "glorieuse ascendance", à l'enseigne des "champs d'honneur". Mais cette ironie a ceci de particulier qu'elle n'est en rien décapante, elle voisine au contraire avec l'humour et laisse transparaître une tendresse pudique et réservée qui, jointe à l'extrême sobriété de l'instance énonciative du récit, m’a suggéré cette réflexion sur laquelle je voudrais conclure.

La psychanalyse nous enseigne qu'il n'y a pas de mot pour dire l'attachement à la mère, l'amour pour le père, mêlés de haine et de culpabilité; le langage fait défaut quand il s'agit de soutenir l'ambivalence sur laquelle ces sentiments reposent, irrésistiblement entraînés par le refoulement. Ironie et pudeur, dont l'équilibre aurait pu être précaire, conjuguent ici leurs effets et donnent une forme esthétique à cette confusion des sentiments. De plus, le narrateur absent des romans de Rouaud parvient à suggérer, grâce à un travail sur la forme remarquablement mấtrisé, que l'obligation de passer par le langage impose à l'humain une distance impossible à combler, un vide indicible. Comme s'il reconnaissait la vanité de l'entreprise, il renonce d'emblée au je qu'une narration de la mémoire appelle "naturellement" (à plus forte raison quand elle ouvertement reconnue comme construite à partir d'éléments autobiographiques ${ }^{8}$ ), allant même jusqu'à se camoufler, à l'instar du narrateur de La modification, derrière un vous qui interpelle le lecteur directement:

Vous ne sauriez dire combien de temps a passé depuis la chute tragique, quand quelqu'un, vous avez oublié qui, pousse la porte de la chambre et, après un silence, annonce simplement: c'est fini.

Lexpression est vague et pourrait s'adapter à mille situations. Pourtant, spontanément, vous comprenez qu'en ce vingt-six décembre mille neuf cent soixante-trois, à l'âge de quarante et un ans, votre père vient de mourir (HI, p: 114).

8 - Ce retour à Random, "chez nous", chez les R'ouaud, s'opère une génération plus tard. [Il s'agit Des hommes illustres.] Après le destin des grands-parents, lié à la Première Guerre mondiale, l'écrivain de la mémoire poursuit le roman de sa famille, (tome 2 ?) centré ici autour du rescapé d'entre tous ces morts, ce "prodigue de vie", le nommé Joseph, père de l'écrivain. Valérie Marin La Meslée, - À la gloire de mon père , dans le magazine littéraire, $\mathrm{n}^{\circ} 315$, novembre 1993 , p. 65. 
76

La narration atteint ainsi à une grande intensité, exprimée avec une extrême pudeur, un détachement qui fait littéralement rebondir l'affect sur le lecteur. Le procédé - bien que la différence de ton soit considérable, cela va sans dire - n'est d'ailleurs pas sans rappeler le roman de Robbe-Grillet où un narrateur absent réussit à induire la jalousie sans jamais mentionner le sentiment éponyme dont il montre la genèse et l'évolution. Ici, l'ironie, qui accentue ce détachement apparent du narrateur, n'en permet pas moins à une grande tendresse de poindre, une tendresse qui devient en quelque sorte effet de lecture.

Le résultat est d'une efficacité déconcertante et témoigne d'une maîtrise du verbe qui repose sur une sobriété savamment dosée, des appels au lecteur virtuels judicieusement placés. L'utilisation du présent historique, qui fait participer plus étroitement le lecteur à la scène, et du présent gnomique, qui ajoute au caractère d'universalité de sentiments suggérés, parachèvent l'effet obtenu et justifient ces titres collectifs - Les champs d'bonneur, Des bommes illustres - pour coiffer des récits dont tout indique qu'ils reposent sur des éléments autobiographiques. Car ils ont atteint une forme qui en assure la dimension littéraire, en élevant le particulier à la dignité de l'universel. 